\title{
THE COHOMOLOGY OF COVERING SPACES OF $H$-SPACES
}

\author{
BY WILLIAM BROWDER
}

Communicated by S. Bochner, February 18, 1959

In this note, the cohomology of a covering space of an $H$-space is computed, in terms of the cohomology of the $H$-space, for coefficients in a field. If the characteristic of the field is different from 2, we also calculate the ring structure.

Let $X$ be an arcwise connected space with a continuous multiplication with unit (an $H$-space). We will suppose that $H^{q}\left(X ; Z_{p}\right)$ is finite dimensional for each $q$ (singular cohomology will be used throughout). Let $\bar{X}$ be a covering space of $X, \pi: \bar{X} \rightarrow X$ the covering map, $G$ the group of deck translations of $\bar{X}$ over $X$, i.e. the fibre of $\pi$. Then $\bar{X}$ can be given an $H$-space structure so that $\pi$ is a multiplicative map.

Let us consider the spectral sequence of Leray-Cartan for this covering space. We can obtain it by replacing $X$ by a homotopically equivalent space (again denoted by $X$ ) which is a fibre space over $K(G, 1)$ with fibre $\bar{X}$, the inclusion of $\bar{X}$ in $X$ being homotopic to $\pi$, and the fibre map $f: X \rightarrow K(G, 1)$ being multiplicative. The group $G$ acts trivially on $H^{*}\left(\bar{X} ; Z_{p}\right)$, so we have simple coefficients in $E_{2}$ (with coefficients in $Z_{p}$ ).

TheOREM. Let $p$ be an odd prime. Then $H^{*}\left(\bar{X} ; Z_{p}\right)=A \otimes E$ as rings, where $A=\pi^{*}\left(H^{*}\left(X ; Z_{p}\right)=H^{*}\left(X ; Z_{p}\right) / I, I\right.$ is the ideal generated by $f^{*}\left(H^{*}\left(K(G, 1) ; Z_{p}\right)\right), E$ is the exterior algebra on $n$ generators $x_{1}, \cdots, x_{n}$, where the dimension of $x_{i}=2 p^{r_{i}}-1$ and $2 p^{r_{i}}$ are the dimensions of a system of generators of the kernel of $f^{*}$. If $p=2$, then the same result holds, but only as modules.

The proof rests on the multiplicative nature of the fibre map $f$. This enables us to introduce a diagonal map into the spectral sequence and obtain a spectral sequence of commutative Hopf algebras [1]. Using the fact that $E_{2}=H^{*}\left(\bar{X} ; Z_{p}\right) \otimes H^{*}\left(K(G, 1) ; Z_{p}\right)$ as Hopf algebras, one can show that if $G=Z_{p^{n}}$, then the kernel of $f^{*}$ is a polynomial ring with one generator $y$ in dimension $2 p^{r}$, and $d^{2 p^{r}}$ is the only nontrivial differential in the spectral sequence. There is an indecomposable element $x \in H^{*}\left(\bar{X} ; Z_{p}\right)$ such that $d^{2 p^{r}}(x)=y$, and thus $x$ is not in the image of $\pi^{*}$. If $G=Z$, then all $d^{m}$ 's are trivial and $E_{2}=E_{\infty}$, (a result due to Serre [2]), while if $G=Z_{q}, q$ prime to $p$, then $H^{*}\left(K(G, 1) ; Z_{p}\right)=0$ and $H^{*}\left(\bar{X} ; Z_{p}\right)$ is isomorphic to $H^{*}\left(X ; Z_{p}\right)$. The 
result in these cases now follows easily with the help of Borel's theorem on the structure of commutative Hopf algebras [3].

For any covering space $\bar{X}$ of $X$ with a group $G$, we may construct a chain of spaces

$$
\bar{X}=X_{n} \stackrel{\pi_{n}}{\longrightarrow} X_{n-1} \stackrel{\pi_{n-1}}{\longrightarrow} \cdots \stackrel{\pi_{1}}{\longrightarrow} X_{0}=X
$$

each $X_{i}$ a covering space of $X_{i-1}$ with fibre a cyclic group, and $\pi_{1} \pi_{2} \cdots \pi_{n}=\pi$. This reduces the problem to the simple cases above.

For $p=2$, there is an ambiguity in the calculation of the ring structure resulting from the fact that the square of an odd dimensional element is not necessarily zero in cohomology mod 2.

If $\bar{X}$ is the universal covering space of $X$, the ideal $I$ is generated by $H^{1}\left(X ; Z_{p}\right)$ and the subspace $B$ of $H^{2}\left(X ; Z_{p}\right)$ consisting of elements which are the images of $H^{1}\left(X ; Z_{p}\right)$ under any Bockstein homomorphism $\beta_{p}$ s. The dimension of the $x_{i}$ 's is determined by writing the algebra generated by $B$ as the tensor product of polynomial rings and truncated polynomial rings. For each truncated polynomial ring on one generator we get one $x_{i}$ whose dimension is the height of the generator minus one.

Corollary. If $H^{q}\left(X ; Z_{p}\right)=0$ for $q>m$, then $H^{q}\left(\bar{X} ; Z_{p}\right)=0$ for $q>m, p$ any prime.

\section{REFERENCES}

1. J. C. Moore, Double suspension and p-primary components, Bol. Soc. Mat. Mexicana vol. 1 no. 1 (1956) pp. 28-37.

2. J. P. Serre, Homologie singulière des espaces fibrés, Ann. of Math. vol. 54 (1951) pp. $425-505$.

3. A. Borel, Sur la cohomologie des espaces fibrés principaux et des espaces homogènes, Ann. of Math. vol. 57 (1953) pp. 115-206.

CORNELl University 